This document is the Accepted Manuscript version of a Published Work that appeared in final form in J. Chem. Phys. 145, 164313 (2016), copyright $(\odot$ American Institute of Physics after peer review and technical editing by the publisher. To access the final edited and published work see

http://aip.scitation.org/doi/10.1063/1.4964845 


\title{
Excess electrons in methanol clusters: beyond the one-electron picture
}

\author{
Gábor Pohl, ${ }^{1}{ }^{\text {Letif }}$ Mones $^{2}$ and László Turi ${ }^{{ }^{*}}$ \\ ${ }^{1}$ Eötvös Loránd University, Department of Physical Chemistry, Budapest 112, P. O. Box
}

32, H-1518, Hungary

${ }^{2}$ University of Cambridge, Engineering Department, Cambridge, CB2 1PZ, United

Kingdom

We performed a series of comparative quantum chemical calculations on various size negatively charged methanol clusters, $\left(\mathrm{CH}_{3} \mathrm{OH}\right)_{n}^{-}$. The clusters are examined in their optimized geometries $(n=2-4)$, and in geometries taken from mixed quantum-classical molecular dynamics simulations at finite temperature $(n=2-128)$. These latter structures model potential electron binding sites in methanol clusters and in bulk methanol. In particular, we compute the vertical detachment energy (VDE) of an excess electron from increasing size methanol cluster anions using quantum chemical computations at various level of theory including a one-electron pseudopotential model, several density functional theory (DFT) based methods, MP2 and $\operatorname{CCSD}(\mathrm{T})$ calculations. The results suggest that at least four methanol molecules are needed to bind an excess electron on a hydrogen bonded methanol chain in a dipole bound state. Larger methanol clusters are able to form stronger interactions with an excess electron. The two simulated excess electron binding motifs in methanol clusters, interior and surface states, correlate well with distinct, experimentally found VDE tendencies with size. Interior states in a

\footnotetext{
*E-mail: turi@chem.elte.hu, fax: (36)-1-372-2592.
} 
solvent cavity are stabilized significantly stronger than electron states on cluster surfaces. Although we find that all the examined quantum chemistry methods more or less overestimate the strength of the experimental excess electron stabilization, MP2, LC-BLYP and BHandHLYP methods with diffuse basis sets provide a significantly better estimate of the VDE than traditional DFT methods (BLYP, B3LYP, X3LYP, PBE0). A comparison to the better performing many electron methods indicates that the examined one-electron pseudopotential can be reasonably used in simulations for systems of larger size. 


\section{Introduction}

Excess electrons solvated in polar solvents are exotic species that have captured the interest of scientists in a wide range of fields for more than 100 years. It is undisputable that the aqueous case, the hydrated electron, has a distinguished role in this group, ${ }^{1}$ but other cases also possess interesting physical properties. A prominent example is the solvated electron in the simplest alcohol, methanol. Since methanol has become a popular target in fuel-cell research (methanol economy), ${ }^{2}$ redox reactions of methanol have received a wider attention in the last decade. Understanding the chemical reactivity of methanol molecules with charged elementary particles (electrons and protons) has a central role in this potentially important field, that points far beyond the challenge of characterizing and interpreting the physical properties of solvated electrons in methanol.

Experiments performed for more than 50 years, have successfully characterized the structure, the dynamics and spectroscopy of solvated electrons in bulk methanol., $3,4,5,6,7,8,9,10$ Extension of the experimental techniques to the gas phase has made it possible to investigate solvated electrons in methanol clusters in the last decade. ${ }^{11,12,13}$ Due to the fewer degrees of freedom of the solvating molecules, cluster experiments provide more tangible information on the underlying microscopic details of the solute-solvent interaction and dynamics. ${ }^{14}$

Ultrafast photoelectron imaging technique revealed two distinctly different, sizedependent trends of the vertical detachment energy (VDE) of the excess electron in methanol clusters. ${ }^{11,12}$ The common interpretation of the data suggests the presence of two isomer classes in experiments, the more strongly bound feature indicating internally solvated excess electrons (interior-bound states), the more weakly bound states signaling surface-bound excess electrons. ${ }^{11,15}$ In comparison, water cluster experiments indicate at least three different excess 
electron binding patterns. ${ }^{16,17}$ Nevertheless, both water and methanol cluster experiments clearly show that the experimental signals strongly depend on the cluster preparation conditions. ${ }^{11,16}$

Theoretical investigations of the electron solvation have focused mainly on the aqueous case. Excess electrons in methanol have been studied using static density functional theory calculations with modest basis sets some time ago. ${ }^{18}$ One-electron quantum-classical molecular dynamics (QCMD) simulations were also used to examine the dynamics of electron solvation in methanol. ${ }^{19,20,21,22,23,24,25}$ Although good general agreement was reached for the physical properties of the solvated electron in bulk methanol, inaccuracies in the simulated optical absorption spectrum, and the publication of new experimental results for methanol cluster anions motivated a development of an improved electron-methanol molecule interaction potential. ${ }^{26}$ The improved potential was then successfully applied in QCMD simulations of excess electrons in bulk methanol and in methanol clusters..$^{26,27,28,15}$ The emerging molecular picture provided semiquantitative agreement between experiment and theory, and in particular, was consistent with the presence of two isomers in cluster experiments. ${ }^{11,15}$ Nevertheless, despite the relatively clear picture of the mechanistic details of the interactions and the dynamics, it is surprising that no effort has been made to perform high level quantum chemistry and many-electron quantum dynamics calculations on methanol cluster anions. The aqueous case that has been thoroughly investigated highlights the essentially trivial statement that careful benchmarking is necessary to critically examine and characterize the applicability of the available methods..$^{29,30,31,32,33}$ It was found that a) at least MP2 level calculations and diffuse basis functions are required to reach reasonable description of the hydrated electron system; b) density functional theory based methods can be utilized only with sufficient care and after careful benchmarking, and c) carefully parameterized one-electron pseudopotentials may provide a semi-quantitative picture of the 
phenomenon and, thus, their application may be advantageous relative to DFT calculations, in particular, in large, non-reactive systems. ${ }^{33}$

Clearly, similar treatment is necessary for methanol if one wants to go beyond the oneelectron-approach dictated simple picture. Such treatment is the purpose of the present and subsequent papers. In the first step we examine the applicability of several density functional theory (DFT) functionals and an electron-methanol molecule interaction potential for the solvated electron in methanol. Configurations of increasing size are generated using one-electron QCMD trajectories. This procedure is identical to that we applied in our hydrated electron studies, ${ }^{33,34}$ and its aim is to generate realistic methanol configurations that model potential binding sites for the excess electron. Comparative calculations of the electron vertical detachment energy are carried out on the pre-formed binding sites of the cluster configurations that confine the electron in an interior solvent cavity, or bind the electron on the surface of the cluster. The motivation for such a comparison is to find a suitable method for many electron molecular dynamics simulations, also one of the aims of our methanol project. These studies may hopefully lead to realistic simulations of electron initiated reactions in methanol.

The structure of the paper will be as follows. In Sec. II, we briefly describe the main features of the QCMD techniques used to generate the configuration sets, and the quantum mechanical computational techniques that are employed in the present study. Sec. III collects the results of the benchmark calculations followed by a systematic analysis and comparison of the results. Sec. IV provides a discussion and concludes the paper.

\section{Methods}


In the present paper we performed a combination of one-electron mixed quantum-classical simulations and various level quantum chemistry calculations. The one-electron simulations are used to generate cluster configurations of different size that, in turn, are analyzed in manyelectron computations. As a prelude for the fixed geometry calculations extracted from the QCMD trajectories, we also optimized small neutral and negatively charged methanol clusters using quantum chemistry calculations for benchmarking purposes of the applied methods and basis sets. Here, neutral $\left(\mathrm{CH}_{3} \mathrm{OH}\right)_{2}$ and negatively charged $\left(\mathrm{CH}_{3} \mathrm{OH}\right)_{2-4}^{-}$clusters were subjected to full geometry optimization and frequency calculation.

We produced equilibrium QCMD trajectories of canonical ensembles at $200 \mathrm{~K}$ for $\left(\mathrm{CH}_{3} \mathrm{OH}\right)_{n}^{-}$clusters for the $n=128$ case with an interior state excess electron, and for the $n=50$ cluster with a surface-bound excess electron. For both methanol cluster anions 100 configurations were selected from 100 ps long equilibrium trajectories, evenly separated by 1 ps time segments. The principles of the QCMD simulations are well documented. ${ }^{35}$ The actual implementation of the QCMD technique is identical to that described in Ref. 15. We note that the simulation uses a flexible all-site methanol classical potential, ${ }^{36}$ and an electron-methanol molecule pseudopotential. ${ }^{26}$ The present model is non-polarizable, but includes polarization in an average, spherically symmetric way. This interaction potential was successfully employed to identify two different, experimentally seen tendencies of VDE of methanol cluster anions with size. Nevertheless, it was observed that the VDE values are too strong relative to the experimental data. The model also provided a detailed molecular picture of a possible relaxation channel following electron attachment to neutral methanol clusters. ${ }^{15}$ 
Of the QCMD generated trajectories we created smaller clusters of various size that were investigated using quantum chemistry methods. The small clusters were generated similarly to our previous aqueous analysis. ${ }^{33} \mathrm{We}$ determined the center of mass of the solvated electron using the one-electron pseudopotential, and produced clusters of gradually increasing size consisting of $n$ methanol molecules $(n=2-8)$ that are nearest to the center of mass of the electron. These clusters are thought to model the first (and part of the second) solvation shell of the excess electron. We note that we distinguish two types of structures, interior and surface state structures. These names, however, refer only to the electron binding motifs of the QCMD simulated parent clusters. The excess electron binding character of the smaller investigated structures is not necessarily identical to that of the corresponding large cluster. In the interior configurations the methanol molecules surround a cavity with their $\mathrm{OH}$ bonds pointing toward the cavity. This arrangement is similar to that seen in interior structure water cluster anions. ${ }^{33}$ In surface state configurations dangling $\mathrm{OH}$ hydrogens localize the excess electron. We then performed various level quantum mechanical calculations on these clusters (100 configurations for each size), and calculated the energy difference between the anionic and the neutral species. This quantity is equivalent to the energy needed to remove the electron from the anion vertically, without changing its nuclear geometry, the vertical detachment energy. All the data (geometries and energies) can be found in the Supplementary Material.

The applied quantum chemistry methods included coupled-cluster $\operatorname{CCSD}(\mathrm{T}),{ }^{37}$ secondorder MP2 perturbation theory, ${ }^{38}$ and DFT methods using the BLYP, ${ }^{39,40}$ LC-BLYP, ${ }^{39,40,41}$ B3LYP ${ }^{40,42,43}$ BHandHLYP, ${ }^{44,45}$ PBE0, ${ }^{46,47}$ X3LYP, ${ }^{48}$ M062X ${ }^{49}$ and $\omega_{B} 97 X^{50}$ functional models. The BLYP and B3LYP functionals are widely used for studies on hydrogen bonded systems. The BLYP functional was found effective in $a b$ initio molecular dynamics (AIMD) 
simulations on water. ${ }^{51}$ The performance of various DFT methods on negatively charged water clusters was also investigated by other studies. It was found that B3LYP and PBE0 perform better than BLYP, but BLYP still gives reasonable results. ${ }^{52}$ Later studies indicated that even B3LYP and PBE0 overestimate electron binding ${ }^{33}$ but using the BHandHLYP functional provides significantly better agreement with MP2 data. ${ }^{29,30,33}$ It was pointed out that increasing the Hartree-Fock (HF) character in the exchange part of the functional improves the performance of DFT methods. ${ }^{29,30}$ The lack of HF character in the exchange part of the BLYP functional, and the low HF exchange character in B3LYP and PBE0 methods results in poor description of the long-range interactions. ${ }^{29}$ Since the BHandHLYP functional employed in present work has much higher HF exchange character than B3LYP or PBE0, better description of the long-range interactions can be expected. The present work employs Becke's half and half functional (BHandHLYP) with the modifications introduced in Gaussian09. ${ }^{45}$ The lack of the description of the long-range interactions in BLYP (and other generalized gradient approximation (GGA) based functionals) can also be improved by using the long-range correction (LC) scheme of Iikura et $a l .{ }^{41}$ Hybrid GGA functionals (such as B3LYP or BHandHLYP) cannot be combined with this particular LC procedure, since the LC part is developed only for pure GGA functionals (e.g. BLYP). ${ }^{41}$ We also note that the LC-BLYP method is a single case of the LC- $\mu$ BLYP procedures with a particular choice of the range separation parameter $\left(\mu=0.47 \mathrm{bohr}^{-1}\right) .^{41,53}$ Tuning $\mu$ is a potential way to improve the capabilities of the LC- $\mu$ BLYP methods ${ }^{54}$ The ab initio MP2 method is also widely used for studies on charged clusters because of the reliable description of exchange and correlation, and moderate resource requirements. ${ }^{55}$ However, it was shown that the performance of MP2 worsens for small systems or for systems with weakly bound electrons. ${ }^{29,30}$ 
Herbert and coworkers studied the basis set dependency of VDEs calculated for negatively charged water clusters. They found that a) utilizing triple zeta over double zeta basis functions resulted only in small improvement, b) adding polarization functions to heavy atoms improved the results, but adding polarization functions to hydrogen atoms led only to marginal improvement, and c) adding more diffuse functions on heavy atoms and hydrogens significantly improved the results. ${ }^{29}$ The basis sets used in the present study were $\operatorname{aug} N$-cc-pVXZ $(N=3,4$ and $\mathrm{X}=\mathrm{D}, \mathrm{T})$ and $6-31(1+3+) \mathrm{G}(\mathrm{d})$. The $6-31(1+3+) \mathrm{G}(\mathrm{d})$ basis set $^{29}$ was created by adding two additional diffuse $s$ orbitals on the hydrogen atoms to the $6-31++\mathrm{G}(\mathrm{d})$ basis set. ${ }^{56}$ The $\operatorname{aug} N$-ccpVXZ basis $\operatorname{set}(N=3,4, \mathrm{X}=\mathrm{D}, \mathrm{T})^{29}$ was built by adding two or three additional set of diffuse $s p$ orbitals on the heavy atoms, and two or three additional diffuse $s$ functions on the hydrogens to the standard aug-cc-pVDZ or aug-cc-pVTZ basis sets. ${ }^{57}$ The exponents of these subsequent diffuse functions were one third of the exponents of the previous one. ${ }^{29}$ We note that the performance and limitations of the $6-31(1+3+) G(d)$ and the aug3-cc-pVDZ sets have been analyzed previously in hydrated electron calculations. ${ }^{29,30}$ In addition, we found the following two aspects more recently: $\left.{ }^{33} 1\right)$ The VDE computed with the $6-31(1+3+) G(d)$ set is usually overestimated (in some cases by $\sim 15 \%$ ) for the more strongly bound water cluster anions relative to aug3-cc-pVDZ basis (regardless of the computational method). 2) The VDE values of MP2/6-31(1+3+)G(d) and MP2/aug3-cc-pVDZ methods show a robust linear correlation with the eom-EA-CCSD/aug3-cc-pVDZ computed values. Nevertheless, MP2/6-31(1+3+)G(d) numbers are underestimated by $\sim 70-80 \mathrm{meV}$ in the lowest VDE regime, while they are overestimated above $\sim 0.5 \mathrm{eV}$ with an intercept of $70.0 \mathrm{meV}$ and a slope of 1.177 .

The calculations were performed with the Gaussian09, ${ }^{58}$ and the CFOUR ${ }^{59}$ program packages. 


\section{Results}

\section{Optimized neutral methanol dimers}

Our first goal was to analyze how the applied DFT methods and basis sets, the diffuse 6$3(1+3+) G(d)$, and the aug3 and aug4 variants of the aug-cc-pVDZ and the aug-cc-pVTZ sets perform on small neutral and anionic methanol clusters (Tables I and II). Neutral methanol dimers were selected first (Table I) as plenty of experimental data are available on dimerization enthalpy. ${ }^{60}$ The experimental results on the dimerization enthalpy vary between -4.3 and -3.2 $\mathrm{kcal} / \mathrm{mol}$, averaging $-3.48 \mathrm{kcal} / \mathrm{mol}^{60}$ The highest level theoretical estimate is $-4.11 \mathrm{kcal} / \mathrm{mol}$ by Umer et. al. ${ }^{61}$ Of the tested methods and basis sets, the BHandHLYP/aug3-cc-pVTZ result (-3.55 $\mathrm{kcal} / \mathrm{mol}$ ) was found to be the closest to the experimental average. All dimerization enthalpies obtained with the $6-31(1+3+) \mathrm{G}(\mathrm{d})$ basis set are outside the experimental range, greater in magnitude than the lowest experimental result except for BLYP/6-31(1+3+)G(d). However, for the BLYP functional this seems to be a result of cancellation of errors, as upon switching to a better basis set, the performance of the BLYP functional deteriorates. Across all methods, switching to the more diffuse aug3-cc-pVDZ and aug3-cc-pVTZ basis sets results in a decrease of the magnitude of the dimerization enthalpy by $1.1 \mathrm{kcal} / \mathrm{mol}$ and $1.3 \mathrm{kcal} / \mathrm{mol}$ respectively. This is most likely the result of the smaller basis set superposition error. Even at this much larger basis sets BLYP and LC-BLYP results are outside the experimental range, while $\omega$ B97X-D, PBE0, B3LYP, and X3LYP methods all give reasonable results. We also note that MP2/aug3-cc-pVDZ also predicts dimerization enthalpy outside the experimental bracket by $0.6 \mathrm{kcal} / \mathrm{mol}$. For all methods, the dipole moments of the dimers are found to be between 2.6 and 3.0 Debye which 
indicates an affinity to form negatively charged methanol oligomers with dipole bound electrons. ${ }^{62}$ To collect more information on the performance of these computational methods, methanol oligomer anions are studied below.

\section{Optimized negatively charged methanol clusters}

We performed a full conformational search on $\left(\mathrm{CH}_{3} \mathrm{OH}\right)_{2-4}^{-}$clusters. Three possible conformational groups were found for small methanol oligomers: cyclic, linear and bifurcated. The cyclic structures are the most stable of all, but they show no electron binding capacity due to the lack of free hydroxyl group and/or not having large enough dipole moments. Bifurcated structures have very low stability and were omitted from further investigations. These observations are identical for the trimers and tetramers. Since our goal is to characterize the performance of different methods in describing electron binding, it is necessary to find systems that actually bind the electron. For this reason, the present section focuses on the electron binding capabilities of the linear structures. These structures possess a free hydroxyl group and a large dipole moment so they are ideally suited to bind excess electrons in a dipole bound state. Figure 1 illustrates the optimized linear structures of methanol cluster anions for $n=2,3$ and 4 . Unlike neutral methanol clusters, there are no experimental (or high level calculated) data on methanol cluster anions in this size range. In fact, to the best knowledge of the authors, up to now there has been only one quantum chemical study in the literature on methanol cluster anions. ${ }^{18}$ Here we performed VDE calculations on $\left(\mathrm{CH}_{3} \mathrm{OH}\right)_{2-4}^{-}$anions using various DFT and ab initio methods. Table II collects the computed data. 
In order to have relatively high level benchmark numbers, full optimization and frequency calculation at the BHandHLYP/aug3-cc-pVDZ level of theory was performed on the $\left(\mathrm{CH}_{3} \mathrm{OH}\right)_{2}^{-}$ dimer followed by single point calculations at the CCSD(T)/aug3-cc-pVDZ and CCSD(T)/aug4cc-pVDZ levels of theory. We preferred the BHandHLYP reference geometry based on our finding of the previous section, the ability of the BHandHLYP method to reliably approach the experimental heat of dimerization of the neutral methanol dimer. For a comparison, we also computed the VDE on the optimized LC-BLYP/aug3-cc-pVDZ dimer geometry, as well. Although one anticipates that additional increase of the diffuse character of the applied basis sets may further stabilize the anion relative to the neutral dimer, the essentially zero VDE numbers indicate that electron binding is not expected for the methanol dimer (Table II). Comparing the DFT based VDE results of the dimer anions to the VDE obtained at the $\operatorname{CCSD(T)~level~it~is~}$ apparent that functionals without considerable HF exchange character (B3LYP, X3LYP, BLYP and PBE0 - referred to as 'traditional functionals' from herein) overestimate electron binding by $\sim 100 \mathrm{meV}$. Upon switching to a larger basis set (aug3-cc-pVTZ) the errors slightly increase. Despite performing well on neutral methanol dimers these functionals are clearly not suitable to precisely describe systems with weakly bound electrons. Functionals with large enough HF exchange character (LC-BLYP and BHandHLYP - referred to as 'long-range corrected functionals' from herein) give more reasonable VDE values. MP2 performs similarly reliably, with larger basis sets essentially reproducing the $\operatorname{CCSD}(\mathrm{T})$ numbers.

Before discussing the trimer and tetramer cases in detail, we note that we performed the geometry optimization of the trimers (and tetramers) with the 6-31(1+3+)G(d) basis set only and all larger basis set calculations were carried out on the $6-31(1+3+) G(d)$ optimized geometries. Calculations on the negatively charged dimers showed that increasing the basis set had only a 
negligible effect on the optimized geometries, it only affects the single point energies. This also applies for the $\operatorname{CCSD}(\mathrm{T})$ single point benchmark calculations (Table II). The best estimates for the VDEs are $-11 \mathrm{meV}$ and $5 \mathrm{meV}$ for the trimer, and $56 \mathrm{meV}$ and $60 \mathrm{meV}$ for the tetramer, with the aug3-cc-pVDZ and aug4-cc-pVDZ sets at the BHandHLYP/6-31(1+3+)G(d) geometry, respectively. These results still imply essentially zero binding for the trimers. The smallest methanol oligomer that is unquestionably predicted to be capable of weak excess electron binding is the tetramer. At the tetramer level one also observes that the diffuse character of the basis set is reasonably (within couple of ten meV) converged. In addition, a comparison of the CCSD(T) calculations on the BHandHLYP and LC-BLYP geometries indicates a $\sim 10-20$ meV uncertainty of the VDE with respect to the chosen reference geometry. As for the trends of the capabilities of the DFT methods to reproduce high-level trimer (and tetramer) VDEs, we observe similar behavior as for the dimer anions. Traditional functionals tend to overestimate the excess electron binding even more for the trimers (by $\sim 100-150 \mathrm{meV}$ ) than for the dimers, while the examined long-range corrected functionals give reasonable results with BHandHLYP and LC-BLYP functionals yielding the best agreement with $\operatorname{CCSD}(\mathrm{T})$ data. Interestingly, while LC-BLYP nearly agrees with the $\operatorname{CCSD}(\mathrm{T})$ data, BHandHLYP overestimates them by $\sim 30 \mathrm{meV}$ at the largest examined basis sets. The dispersion corrected $\omega$ B97X-D produces similar agreement with the $\operatorname{CCSD}(\mathrm{T})$ data. Since traditional functionals proved to be the least adequate to describe methanol systems with a weakly bound electron, we calculated the VDEs of the tetramers using only the long-range corrected functionals. The trends for the LC-BLYP, the BHandHLYP and the $\omega B 97 X-D$ methods remained similar as seen for the trimers. We also observe that the VDE values for the tetramers are basically independent of the basis sets (using the same method). 
Based on the observations above, we conclude that of the examined DFT methods LCBLYP, BHandHLYP and $\omega \mathrm{B} 97 \mathrm{X}-\mathrm{D}$ are the most suitable to describe electron binding of an excess electron to small methanol oligomers. As an additional comparison, we note that MP2 performs rather reliably, underestimating the $\operatorname{CCSD}(\mathrm{T})$ tetramer VDE only by $\sim 10 \mathrm{meV}$. Here we remind that up to this point in the paper we have considered only systems with no or very weak electron binding character. Based on the findings for the aqueous case, ${ }^{29,32,33}$ the performance of the MP2 method is expected to improve for larger systems (i. e. with increasing excess electron binding energy).

\section{Solvated electron models up to the first solvation shell}

To test further the efficiency of these methods single point calculations were performed on models of the first solvation shell of an excess electron in methanol. 100 different configurations of a 50 membered methanol cluster anion with a surface bound excess electron and a 128 membered methanol cluster anion with an interior bound electron were taken and studied from QCMD trajectories. We inspected 2 - 8 methanol molecules of these configurations from the first (and second) solvation shell of the solvated electron (i.e. nearest to the center of the excess electron). Illustrative hexamer examples of the two types of structures are shown in Figure 2. In the interior configurations non-hydrogen bonding $\mathrm{OH}$ hydrogen atoms point toward the cavity of the cluster and stabilize the excess electron. The number of free hydroxyl hydrogens in the analyzed interior cases varies between 3 and 6 . This number is six in the selected example (Figure 2, top). In the surface bound configurations, dangling hydrogens, pointing outside the cluster, localize the solvated electron. One can recognize that in most cases the methanol molecules with the dangling hydrogens participate only in a single hydrogen bond, as single 
acceptors (A type). Typically hydrogen bonded chains are formed in the clusters, as emphasized in Figure 2 with the dashed lines. This structural pattern is significantly different from that of the aqueous case where acceptor-acceptor (AA) type water molecules with two dangling hydrogen atoms form the dominant electron binding motif. ${ }^{63,64}$ For methanol, however, only one hydroxyl hydrogen atom is available for each methanol unit to bind directly to the electron. This results in much weaker electron binding and more diffuse excess electron states in surface state methanol cluster anions than in surface state hydrated electron clusters.

In this section we analyze the VDE values computed using the 6-31(1+3+)G(d) basis set. For systems with surface bound electrons the VDEs for 2 and 3 methanols averaged over 100 configurations agree well with the results of dimer and trimer optimizations (see Table II) using the same method. In the surface bound cases, the methanol molecules nearest to the solvated electron readily form linear dimers and trimers, which are similar to the optimized most stable electron binding systems. As the size of system increases, the VDE monotonically rises with all methods, as shown in Figure 3. Note the weak excess electron binding. The average VDE varies in the 30-300 meV range for eight methanol molecules depending on the method. Of the traditional functionals, we used only the BLYP method in this section, anticipating similar behavior for B3LYP and PBE0. BLYP predicts much stronger binding than MP2, LC-BLYP and BHandHLYP (Figure 3), the same behavior as for small optimized systems. This further demonstrates that the former methods are likely to severely overestimate the electron binding for these systems. Of the latter methods, although all three agree reasonably well, we show the results of the MP2 and BHandHLYP methods only. Nevertheless, we find all these methods to be suitable to describe systems with weakly bound surface electrons in methanol clusters. In particular, we note that adding long-range corrections (LC) or increasing the HF exchange 
component (BHandHLYP) greatly improves the performance of the BLYP functional for these systems. Figure 4 shows the correlation between the BHandHLYP and MP2 numbers. The strong correlation agrees well with previous observations for aqueous systems. ${ }^{29,33}$ Nevertheless, one observes that BHandHLYP overestimates the MP2 values by an average $\sim 10 \%$ for most of the configurations, with more significant deviations in the most weakly bound region. Interestingly, while Hartree-Fock calculations slightly underestimate and pseudopotential results mildly overestimate the MP2 VDE's, overall they perform surprisingly well in the investigated weakly bonding surface state region (Figure 3). The weak binding interactions are reflected in the diffuse character of the excess surface electron distributions. We characterize the size of the excess electron using the radius of gyration of the singly occupied molecular orbitals of the anions. The radius of the excess electron falls in the 6 - $12 \AA$ range computed with both BHandHLYP and MP2 regardless of the number of methanol molecules in the clusters.

For systems with interior bound electrons the average VDEs for $n=2-8$ clusters are shown in Figure 5. Examining the trends, one can immediately notice three features of the interior state VDE data: 1) the interior state VDEs are significantly greater than those in surface bound states; 2) the VDE values rapidly increase with system size; and 3) the tendency of the VDE growth is different for the DFT methods and MP2. We discuss this latter aspect in more detail below. The general, significantly overbinding behavior of the BLYP, B3LYP and the PBE0 methods is similar to that seen for the surface bound case (as an example, see BLYP in Figure 5). More moderate VDE values are predicted by the LC-BLYP and BHandHLYP methods. Figure 5 shows only results of the latter method. Once again, it is noteworthy to mention that while the HF method seriously underestimates the VDE, the one-electron pseudopotential does a better job with an $\sim 0.3 \mathrm{eV}$ smaller VDE in average at $n=8$ than MP2. 
We now focus on the comparison of BHandHLYP with MP2. While the average VDE values computed with BHandHLYP increase nearly linearly with cluster size in the examined size range, MP2 results show a slow progress followed by a break and a faster increase after a critical cluster size. A glance behind the average MP2 behavior reveals that for all interior state configurations the first several methanol molecules do not (or only very weakly) bind the electron. Placing a critical number of methanol molecules around the electron suddenly increases the stability of the excess charge. The minimum number of methanol molecules needed to stabilize the electron varies from 4 to 8 molecules. This observation suggests that the presence of nearly the full first solvation shell is necessary for the electron localization and stabilization in methanol. The 100 studied configurations can be divided into subgroups based on the critical electron binding size. Within each of these subgroups the trends in VDE are similar (Figure 6). Once the system reaches the critical size the MP2 and DFT tendencies become similar and the VDE curves run parallel. This similarity is further illustrated in Figure 7, which shows the correlation between the BHandHLYP and MP2 data. The correlation above a $200 \mathrm{meV}$ MP2 computed VDE threshold is linear, similarly to the surface correlation (Figure 4). On the other hand, below $\sim 100 \mathrm{meV}$ practically no correlation exists between the data. In this interaction range BHandHLYP predicts significantly stronger interaction than MP2.

Interestingly, the MP2 trends of the radius of gyration are parallel to those in VDE. Once the cluster reaches the critical electron binding size, the radius of the electron suddenly collapses from its average non-bonding $\sim 8 \AA$ size to approximately 3-4 $\AA$. Adding extra methanol molecules to the cluster further increases the VDE and decreases the radius of the excess electron. BHandHLYP also displays shrinking radius, but we also find very diffuse electron distributions for configurations with positive VDE values up to $200 \mathrm{meV}$. Clearly, the mismatch 
between MP2 and BHandHLYP appears for those configurations that predict diffuse MP2 electron distribution (and weak binding) for the excess electron.

To test the VDE tendencies for the interior electron binding motif at small cluster size, we performed CCSD(T)/aug3-cc-pVDZ calculations on two series of methanol cluster anion configurations that contain an increasing number of methanol molecules with $n=2,3$, and 4 . These configurations were chosen from the 100 possible configurations as a) the one that binds the electron most strongly in the tetramer at the MP2/6-31(1+3+)G(d) level (Configuration A in Figure 8) and b) the one that has the greatest deviation in the tetramer VDE in the BHandHLYP/6-31(1+3+)G(d) and the MP2/6-31(1+3+)G(d) calculations (Configuration B in Figure 8). Figure 8 shows a comparison of the VDE values computed with BHandHLYP/6$31(1+3+) \mathrm{G}(\mathrm{d})$ and MP2/6-31(1+3+)G(d) with the results of $\operatorname{CCSD}(\mathrm{T}) / \operatorname{aug} 3-\mathrm{cc}-\mathrm{pVDZ}$. The $\operatorname{CCSD}(\mathrm{T})$ trend for the most strongly binding tetramer anion is between that of MP2 and BHandHLYP. We observe that the $\operatorname{CCSD}(\mathrm{T})$ points lie somewhat closer to the BHandHLYP values, especially when repeating the MP2 and BHandHLYP calculations with the aug3-ccpVDZ basis set. Figure 8 also contains the data points pertaining to the configuration with the greatest deviation in the MP2 and BHandHLYP calculated tetramer VDE values (-59.2 vs. 235 meV, respectively). The CCSD(T)/aug3-cc-pVDZ calculations predict binding for the trimer (33.2 $\mathrm{meV})$, but non-binding for the tetramer $(-71.9 \mathrm{meV})$ in better agreement with the MP2 numbers. Based on these tendencies, we are inclined to state that BHandHLYP behaves more reasonably in the very weak interior electron binding region than MP2. However, we note that BHandHLYP also finds a relatively strong electron binding for a configuration that is, in fact, non-bonding. Figure 8 also contains the MP2 and BHandHLYP data points for $n=5$, where we find that the excess electron becomes localized for both configurations (VDE > 200 meV). Here 
the VDE values of the two methods correlate better than for smaller clusters (see also Figure 7) and one can expect that both VDE's would approximate the CCSD(T) number reasonably. We note that our previous observation for water cluster anions suggests that MP2 becomes more reliable than BHandHLYP in this more strongly binding region. ${ }^{33}$ Although of the long-range corrected DFT methods we focused on BHandHLYP in the present study, here we reconsider LCBLYP for a moment to demonstrate the effect of optimizing the range separation parameter $(\mu)$ for the case of a single tetramer configuration (Configuration B in Figure 8). LC-BLYP gives similar VDE value to BHandHLYP, $257 \mathrm{meV}$, while MP2 and CCSD(T) predict negative VDE. Optimizing the $\mu$ parameter of LC-BLYP according to the prescription of Baer et al. ${ }^{54}$ leads to a value $\left(0.25\right.$ bohr $\left.^{-1}\right)$ that results in an even greater VDE, $484 \mathrm{meV}$. Although this tendency seems unfavorable, more tests are needed to develop general conclusions on the tuning issue of the range separation parameter in LC- $\mu \mathrm{BLYP}$ for methanol cluster anions.

The last aspect we examine here is the large size tendencies of the VDE. Since none of the examined many electron models can be applied for large methanol cluster anions (i.e. containing several hundred atoms), we focus on the applicability of the one-electron pseudopotential calculations. Figure 9 shows incremental average VDE values computed with the one-electron pseudopotential in large clusters with $n=128$ methanol molecules for both interior bound and surface bound clusters. For a comparison, we also added the VDE values for the $n=128$ cluster anions computed from the experimentally determined linear VDE-size relationship. ${ }^{11}$ The experimental Type I and II data points correspond well to the interior electron binding and surface state electron binding data, respectively. ${ }^{15}$ Clearly, for both cases the pseudopotential slightly overestimates the corresponding experimental data. The inset in Figure 9 contains the data as the function of the inverse cube of the cluster size $\left(n^{-1 / 3}\right)$. The linear behavior for $n>\sim 12$ 
for both surface and interior states demonstrates a good agreement with the prediction of a continuum dielectric theory. ${ }^{65}$

To extend the comparison of our one-electron model and the investigated many-electron quantum chemistry methods in the small-size regime (see Figures 3 and 5) we performed additional series of MP2/6-31(1+3+)G(d) and BHandHLYP/6-31(1+3+)G(d) calculations for 2020 interior and surface state configurations for the $n=12$ clusters. At $n=12$ the MP2, BHandHLYP and pseudopotential average VDE values are 928, 1150 and $711 \mathrm{meV}$ for the interior states, and 86,150 and $84 \mathrm{meV}$ for the surface states, respectively. We found that MP2 and BHandHLYP predict stronger binding for the interior states at larger cluster sizes than the pseudopotential. Although it is difficult to extrapolate the VDE to larger clusters, we can state that the qualitative tendencies and agreement between the data points are promising. This remark is especially valid for the surface state behavior. We note that for the interior states of water cluster anions we observed similar trends with the pseudopotential data overestimating the experimental ones, and underestimating the MP2 values. ${ }^{33}$ The overestimation of the MP2 data relative to experiment may be due to a) the well documented overestimation of the VDE using the MP2/6-31 $(1+3+) \mathrm{G}(\mathrm{d})$ method by $\sim 10-15 \%$, and b) to the fact that the examined pseudopotential generated configurations do not necessarily sample precisely the MP2 configurations space. ${ }^{33}$ Nevertheless, we find that the MP2 (and BHandHLYP) VDE trends further support the assignment of Type I experimental points to interior state clusters and Type II data to surface state clusters.

\section{Discussion and Conclusions}


We performed a series of comparative VDE calculations on various size methanol cluster anions. The applied techniques included a series of DFT methods, MP2, CCSD(T) calculations and a previously developed one-electron pseudopotential method. Interestingly, the calculation of the heat of dimerization of the neutral methanol dimer seriously tested the applicability of the considered methods, although most of the computed data gave a reasonable estimate of the experimentally determined range. The calculation of the vertical detachment energy of an electron from linear methanol anion chains, however, clearly separated the methods. The MP2, the long-range corrected LC-BLYP and BHandHLYP and the dispersion corrected $\omega$ B97X-D methods reasonably reproduced the $\operatorname{CCSD}(\mathrm{T})$ benchmark data (using large diffuse basis sets), while the traditional functionals, PBE0, BLYP, B3LYP, X3LYP significantly overestimated these data. The calculations also indicated that at least four methanol molecules are needed in a hydrogen bonded chain to bind an excess electron in a dipole bound state. We also extended the VDE calculations for larger clusters. The configurations of the clusters were generated from oneelectron mixed quantum-classical simulations. These configurations represented two possible electron binding motifs of the methanol clusters, interior state excess electron configurations and surface state configurations. We found that the better performing quantum chemistry calculations (MP2, BHandHLYP, LC-BLYP) using diffuse basis sets indicate strong binding for interior state configurations, while weak interactions are predicted for surface states. This finding is consistent with a previous assignment based on one-electron pseudopotential calculations suggesting that the experimental Type I clusters are likely to stabilize the electron in the interior of the cluster, while Type II clusters are surface state cluster anions. ${ }^{15}$ A comparison of the computational methods demonstrated that traditional functionals tend to significantly overbind the electron, while long-range corrected functionals (BHandHLYP and LC-BLYP) provide a more reasonable 
estimate of the VDE. We note that further improvement is, in principle, possible by optimizing the range separation parameter of the LC- $\mu \mathrm{BLYP}$ procedures, ${ }^{54}$ as was illustrated in an $a b$ initio molecular dynamics simulation study of the optical spectrum of the hydrated electron. ${ }^{66} \mathrm{We}$ performed tests in this spirit for a small methanol cluster anion. Our preliminary results indicate that the VDE strongly depends on the choice of $\mu$, and the optimal value appears to be system and size dependent. Further work in this direction is under way.

We observed that the tendency of the VDE growth with size is similar in MP2 and the long-range corrected functionals for surface states, but differs slightly for interior states. The performance of the parameter-free MP2 method is promising, in particular in view of the fast developments of the density fitting (or resolution of the identity, RI) MP2 algorithms. ${ }^{67}$ Present implementations of RI-MP2 appear to be comparable in cost to the DFT calculations. ${ }^{68}$ Nevertheless, it remains to be tested whether addition of extra diffuse functions (similar to those used in the present paper) to the auxiliary RI basis sets are adequate. For interior states, $\operatorname{CCSD}(\mathrm{T})$ benchmark calculations confirm that a small number of methanol molecules (at least three) are needed to confine the electron. Once the critical size is reached, the stability of the excess electron quickly grows with cluster size.

\section{Supplementary Material}

See supplementary material for the geometries and energies of the cluster structures examined in the present work.

\section{Acknowledgments}


This work was supported by a research grant to LT from the National Research, Development and Innovation Office, Hungary (NKFIH, previously OTKA, K104237). The authors are grateful to János Sarka (Eötvös Loránd University) for helpful discussions. 


\section{Figure Captions}

Figure 1. Optimized $\left(\mathrm{CH}_{3} \mathrm{OH}\right)_{2-4}^{-}$cluster structures at BHandHLYP/aug3-cc-pVDZ level of theory.

Figure 2. Typical interior (top) and surface (bottom) state structures containing six methanol molecules that are nearest to the center-of-mass of the excess electron in the corresponding MD generated clusters. Spin density contours of the excess electron (enclosing $45 \%$ and $10 \%$ of the excess electron charge density for interior state and surface bound state, respectively) are also shown. The very diffuse surface state excess electron density is truncated to fit the figure.

Figure 3. Average VDE values of various size $\left(\mathrm{CH}_{3} \mathrm{OH}\right)_{n}^{-}$methanol cluster anions $(n=2-8)$ computed with selected quantum chemical methods on surface state configurations taken from the pseudopotential generated QCMD trajectories. The HF, DFT and MP2 calculations were performed using the $6-31(1+3+) \mathrm{G}(\mathrm{d})$ basis set.

Figure 4. Correlation of the vertical detachment energies of $\left(\mathrm{CH}_{3} \mathrm{OH}\right)_{\mathrm{n}}^{-}$methanol cluster anions with $n=2-8$ computed with BHandHLYP/6-31(1+3+)G(d) vs. MP2/6-31(1+3+)G(d). The figure contains configurations that bind the excess electron in surface states.

Figure 5. Average VDE values of various size $\left(\mathrm{CH}_{3} \mathrm{OH}\right)_{n}^{-}$methanol cluster anions $(n=2-8)$ computed with selected quantum chemical methods on interior cavity configurations taken from the pseudopotential generated QCMD trajectories. The DFT and MP2 calculations were performed using the 6-31(1+3+)G(d) basis set.

Figure 6. Average VDE values of various size $\left(\mathrm{CH}_{3} \mathrm{OH}\right)_{\mathrm{n}}^{-}$methanol cluster anions $(n=2-8)$ for those sets of interior configurations where MP2 excess electron binding starts at $n=5$ (red), 6 (black), 7 (blue) and 8 (green). Magenta shows those configurations that are still not bound in the 
octamer configurations. MP2 values are connected with solid lines, while BHandHLYP points by dashed lines. The identical colors for MP2 and BHandHLYP signify averages on the same sets of configurations.

Figure 7. Correlation of the vertical detachment energies of $\left(\mathrm{CH}_{3} \mathrm{OH}\right)_{\mathrm{n}}^{-}$methanol cluster anions with $n=2-8$ computed with BHandHLYP/6-31(1+3+)G(d) vs. MP2/6-31(1+3+)G(d). The figure contains configurations that bind the excess electron in interior states.

Figure 8. Vertical detachment energy of an interior excess electron in two selected molecular dynamics configurations with increasing number of methanol molecules placed around the excess electron's position computed with BHandHLYP/6-31(1+3+)G(d), MP2/6-31(1+3+)G(d) and $\operatorname{CCSD}(\mathrm{T}) / \operatorname{aug} 3-\mathrm{cc}-\mathrm{pVDZ}$.

Figure 9. Incremental VDE values computed in $n=128$ methanol cluster anions using a oneelectron pseudopotential model. Solid squares: interior state clusters; empty squares: surface state clusters. The stars show the VDE values computed from the experimentally determined linear VDE-size relationship for the $n=128$ cluster. $^{11}$ 
Figure 1.
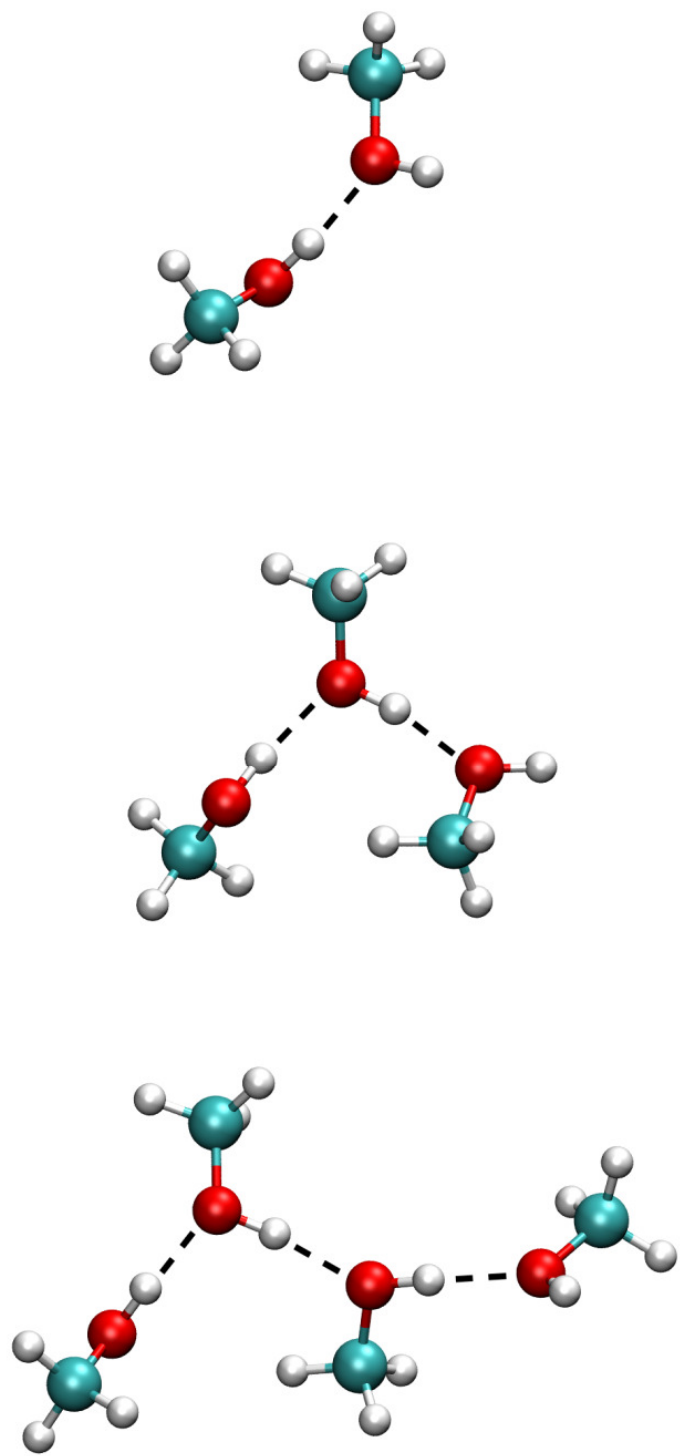
Figure 2.
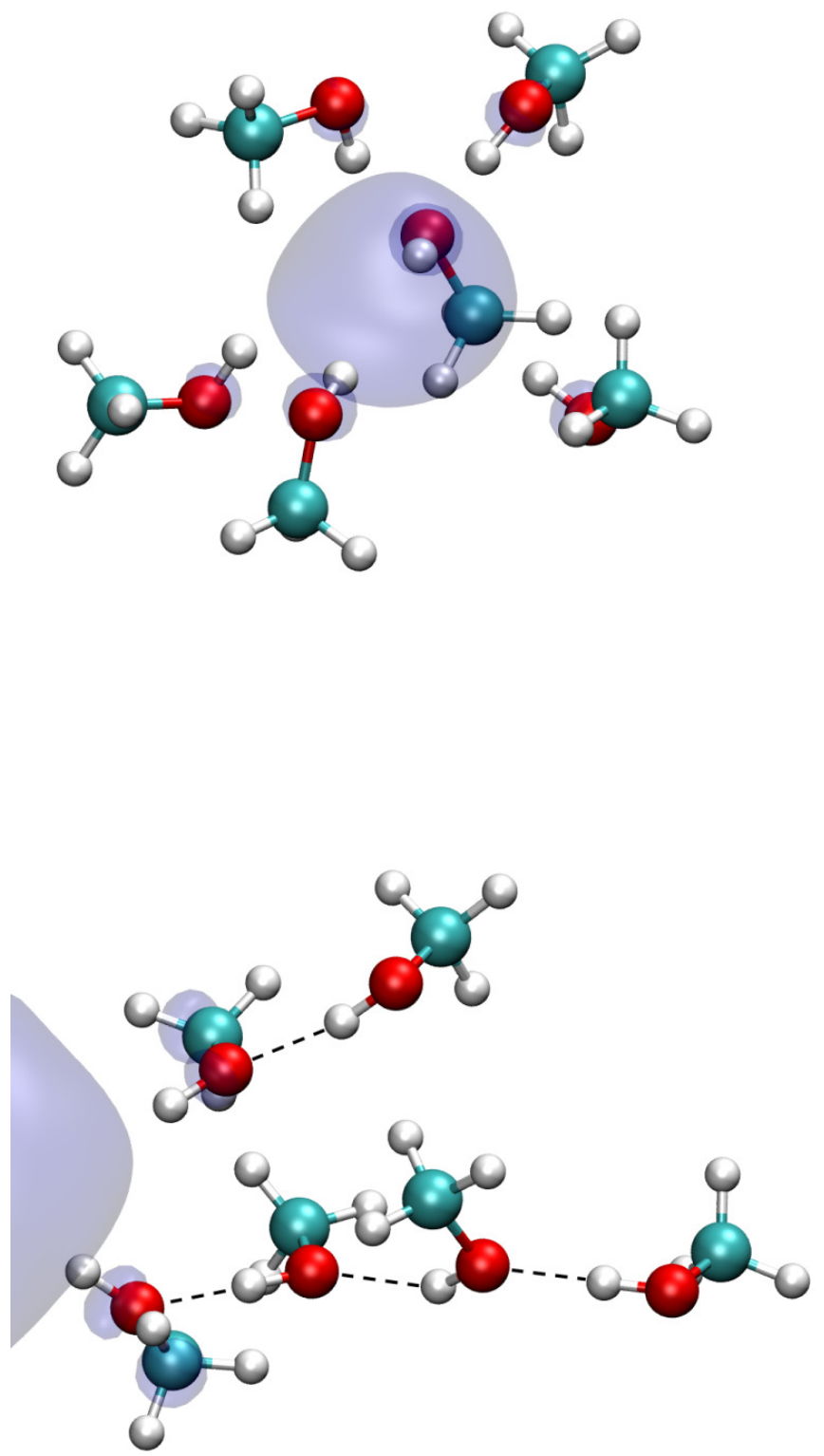
Figure 3.

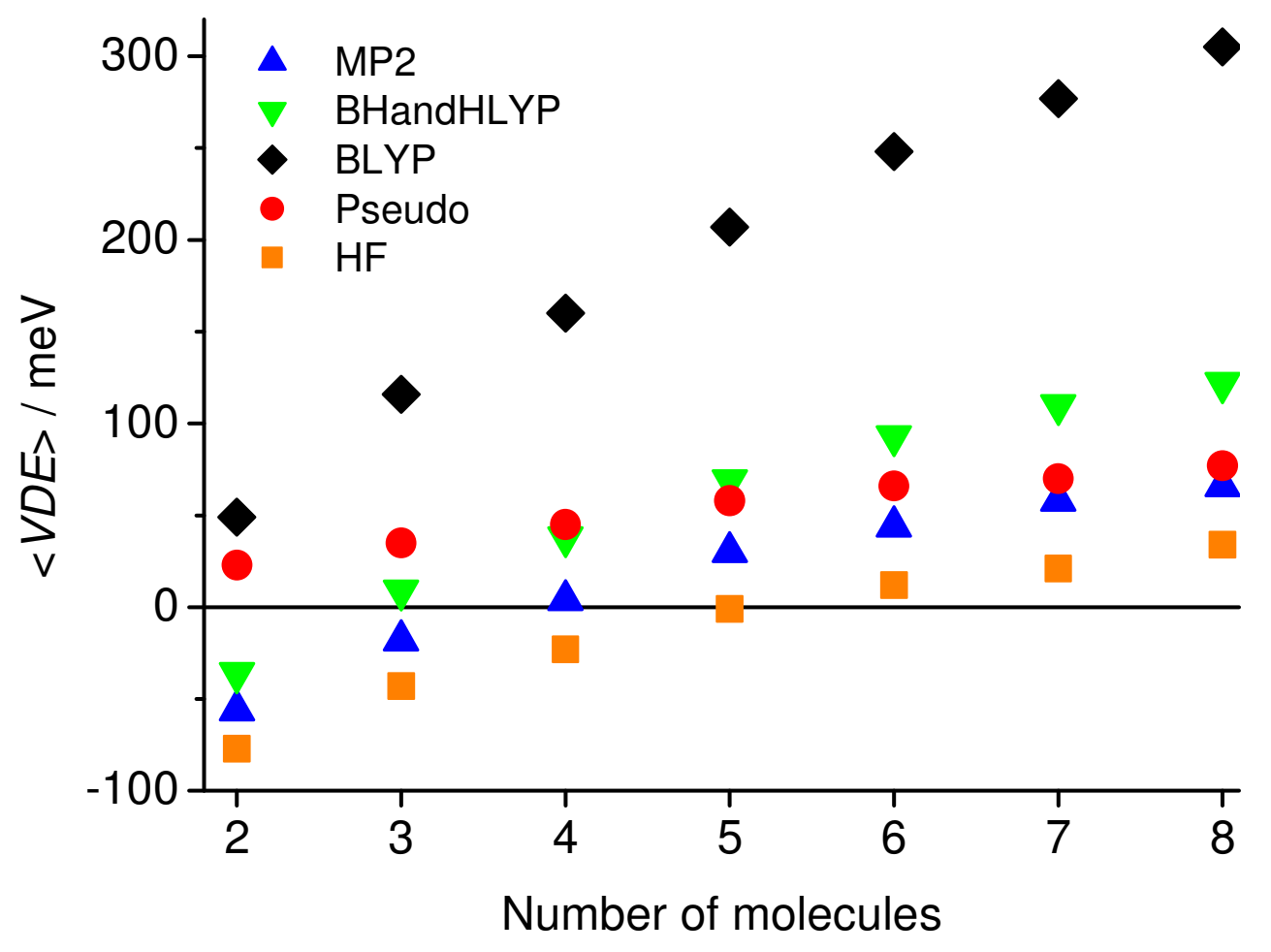


Figure 4.

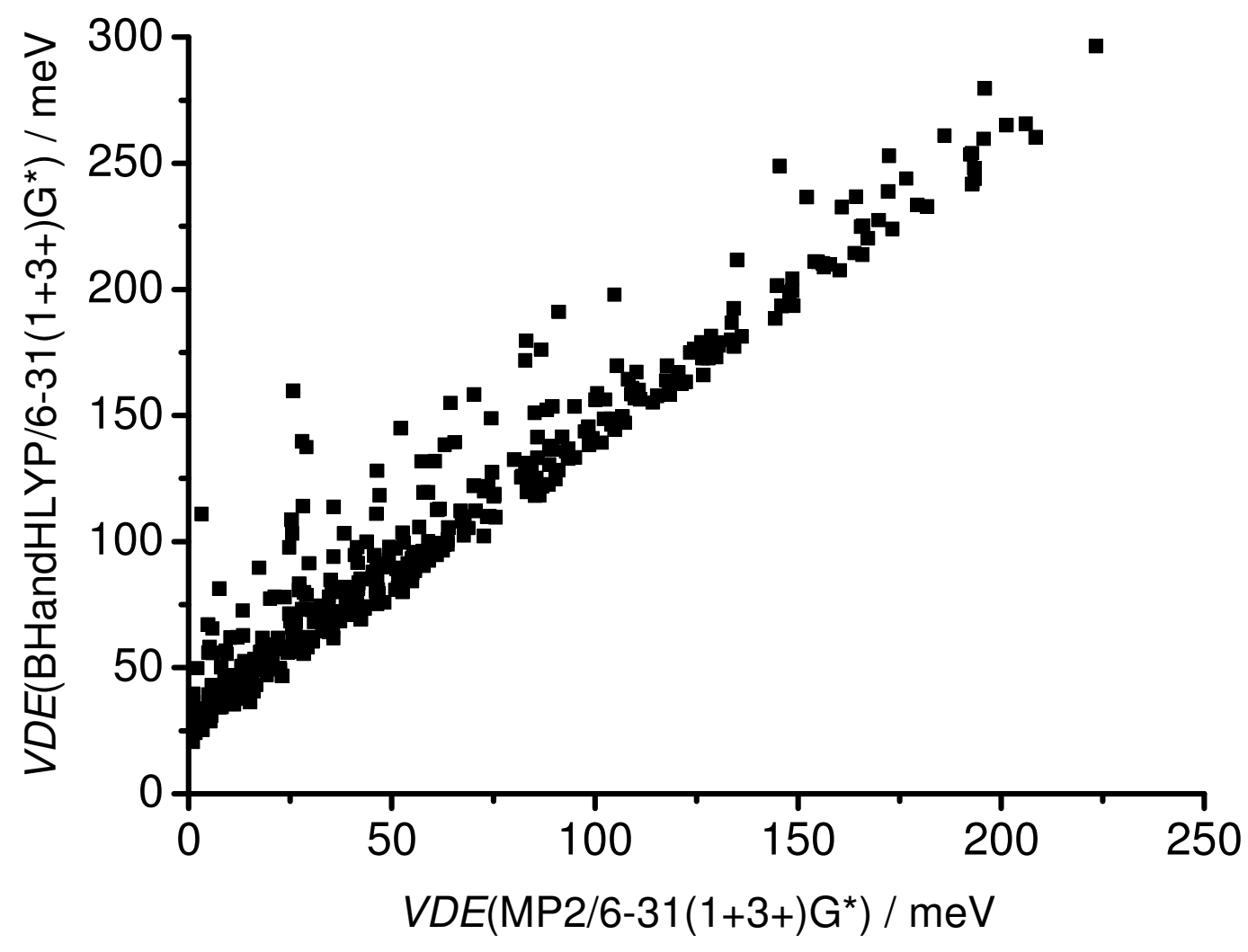


Figure 5.

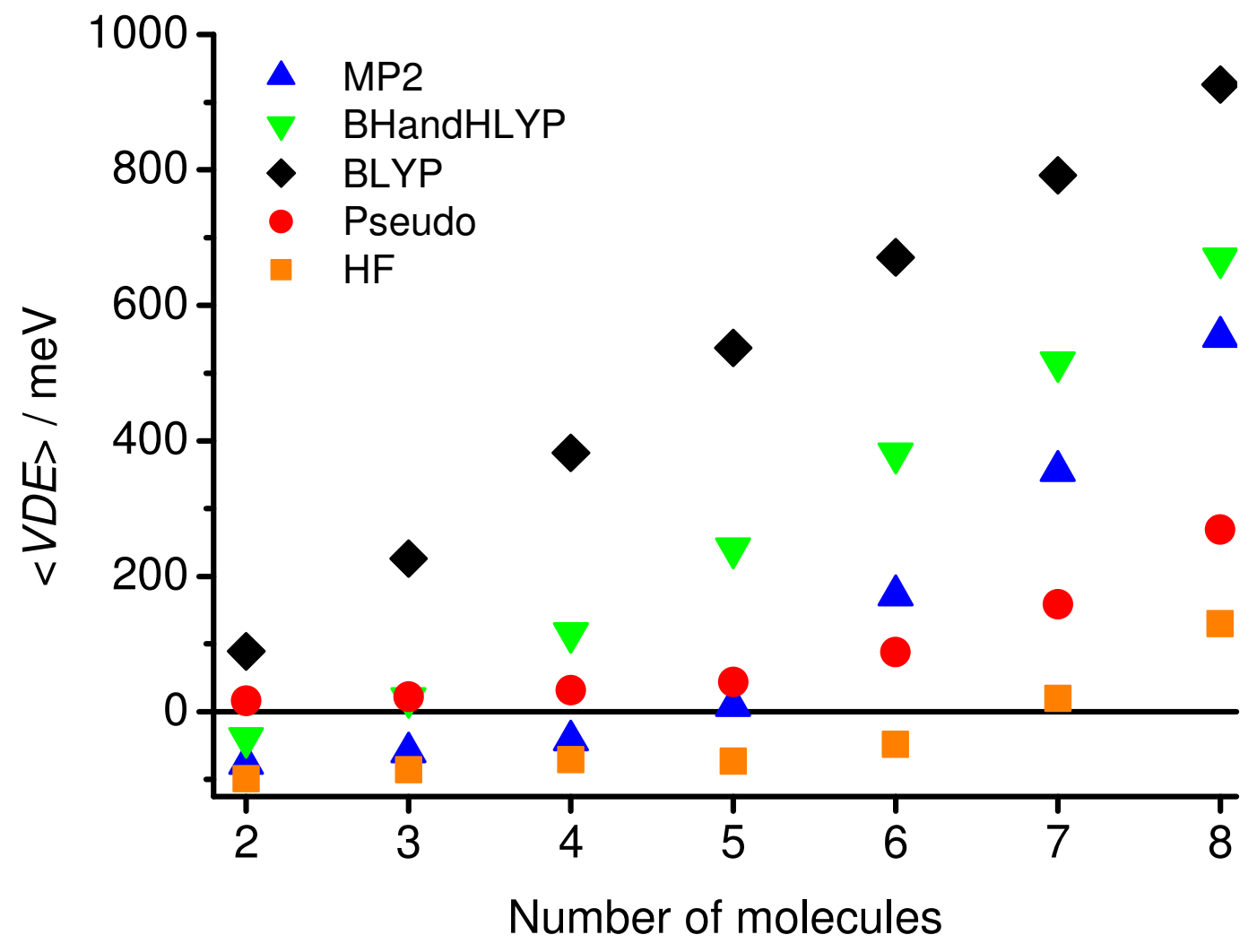


Figure 6.

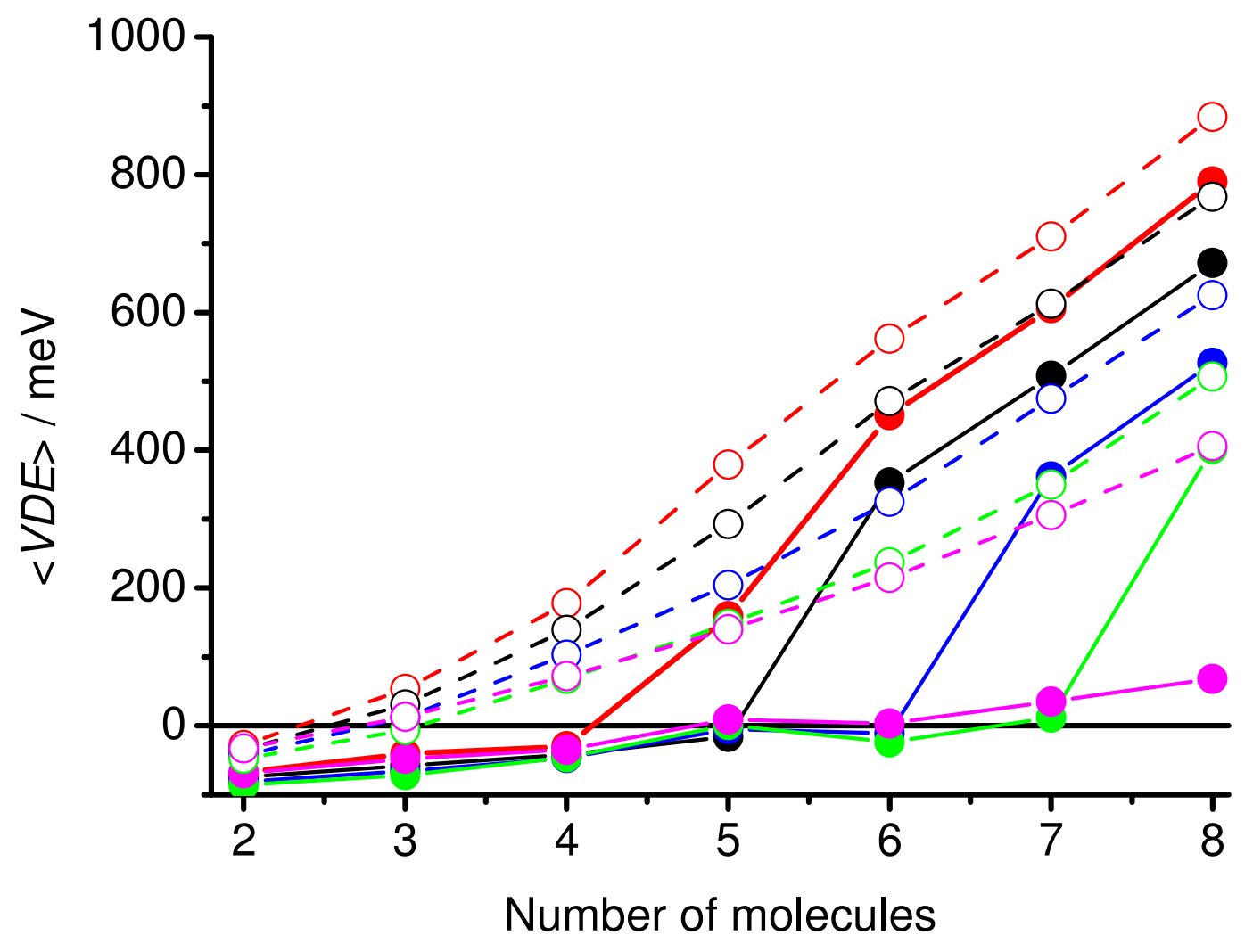


Figure 7.

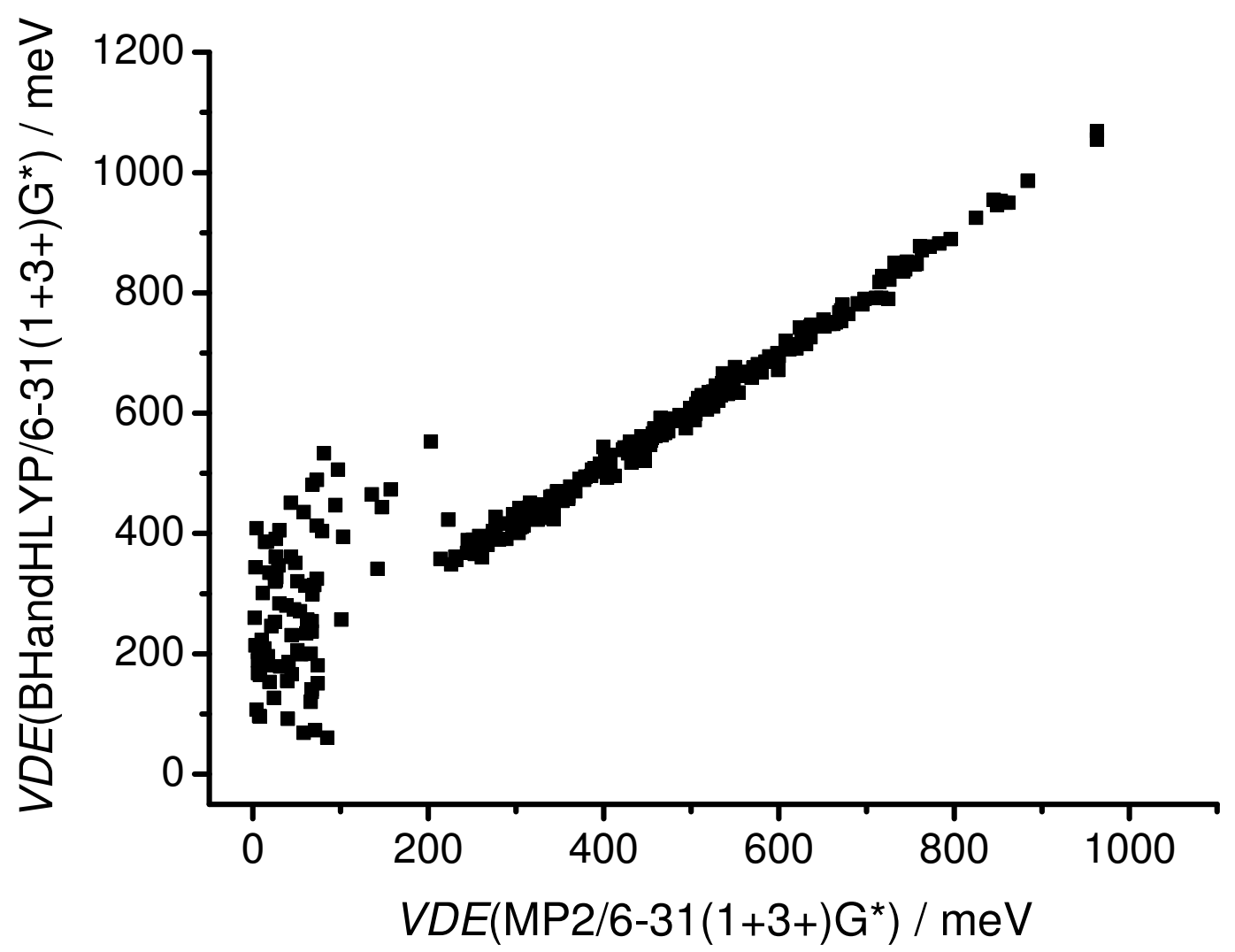


Figure 8.
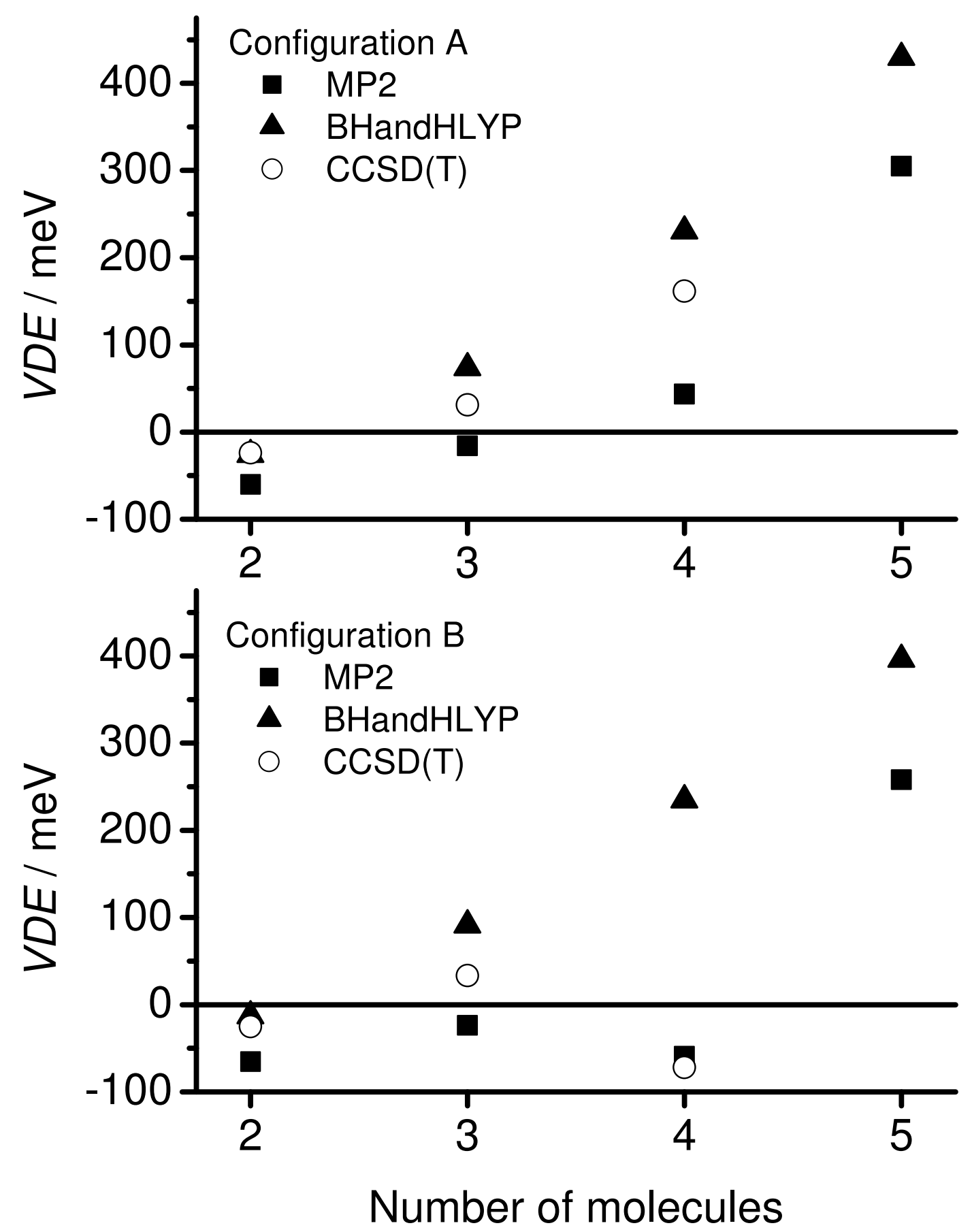
Figure 9.

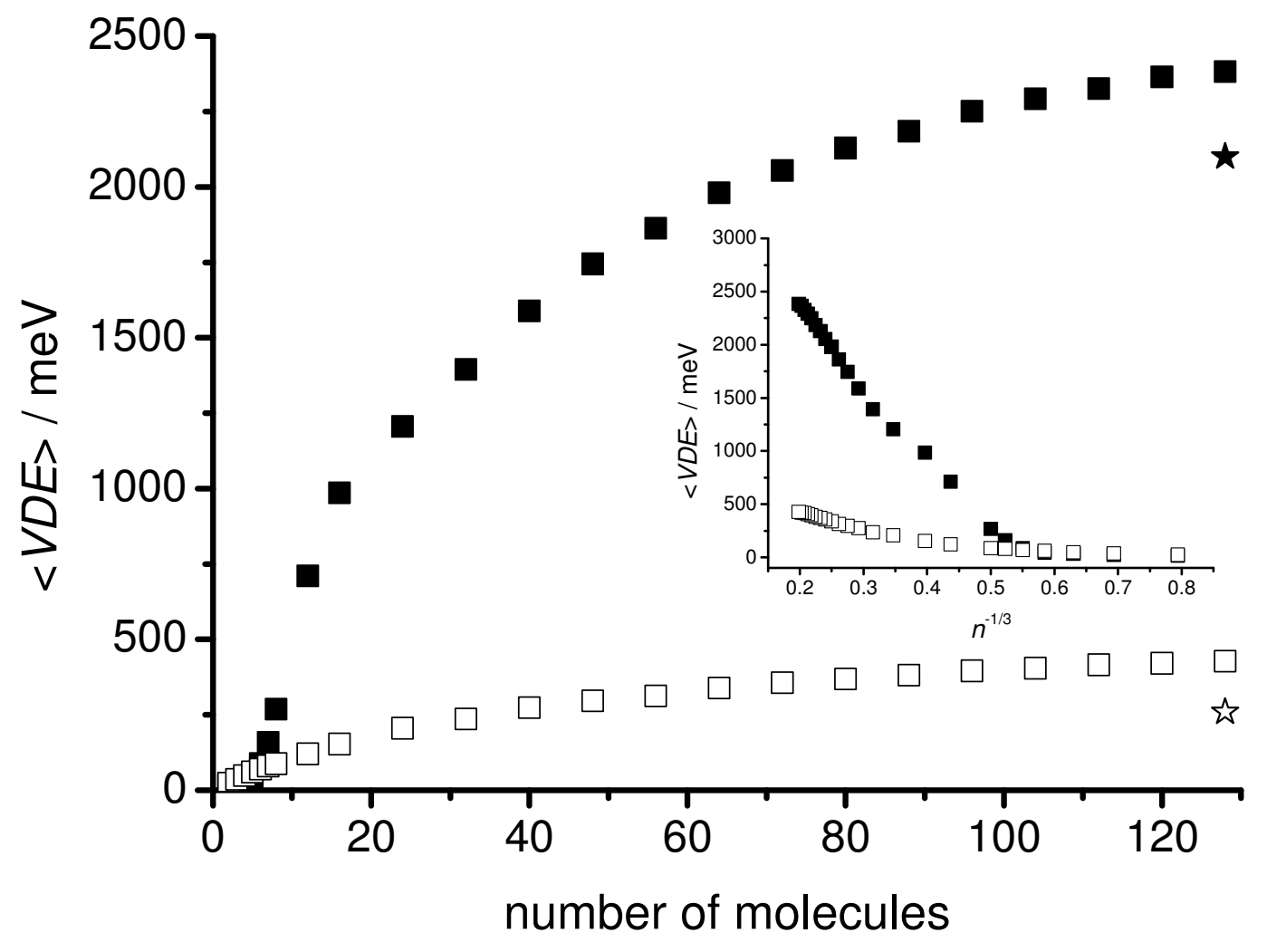


Tables

Table I. Enthalpy of the dimerization and the dipole moment of neutral methanol dimers as obtained at various levels of theory.

\begin{tabular}{|c|c|c|}
\hline Level of theory & $\begin{array}{l}\Delta H_{\text {dimer }} / \\
\mathrm{kcal} / \mathrm{mol}\end{array}$ & Dipole / Debye \\
\hline LC-BLYP/6-31(1+3+)G(d) & -6.62 & 3.24 \\
\hline BHandHLYP/6-31(1+3+)G(d) & -4.93 & 3.01 \\
\hline$\omega \mathrm{B} 97 \mathrm{X}-\mathrm{D} / 6-31(1+3+) \mathrm{G}(\mathrm{d})$ & -5.36 & 2.82 \\
\hline PBE0/6-31(1+3+)G(d) & -5.10 & 2.87 \\
\hline $\mathrm{X} 3 \mathrm{LYP} / 6-31(1+3+) \mathrm{G}(\mathrm{d})$ & -4.95 & 2.93 \\
\hline B3LYP/6-31(1+3+)G(d) & -4.48 & 2.92 \\
\hline BLYP/6-31(1+3+)G(d) & -4.05 & 2.86 \\
\hline$[\mathrm{MP} 2 / 6-31(1+3+) \mathrm{G}(\mathrm{d})$ & -5.98 & $3.06]$ \\
\hline LC-BLYP/aug3-cc-pVDZ & -5.59 & 2.95 \\
\hline BHandHLYP/aug3-cc-pVDZ & -3.77 & 2.82 \\
\hline$\omega B 97 X-D / a u g 3-c c-p V D Z$ & -4.38 & 2.62 \\
\hline PBE0/aug3-cc-pVDZ & -3.89 & 2.66 \\
\hline X3LYP/aug3-cc-pVDZ & -3.83 & 2.73 \\
\hline B3LYP/aug3-cc-pVDZ & -3.39 & 2.73 \\
\hline BLYP/aug3-cc-pVDZ & -3.00 & 2.65 \\
\hline [MP2/aug3-cc-pVDZ & -4.90 & 2.45] \\
\hline LC-BLYP/aug3-cc-pVTZ & -5.29 & 3.05 \\
\hline BHandHLYP/aug3-cc-pVTZ & -3.55 & 3.00 \\
\hline$\omega B 97 X-D / a u g 3-c c-p V T Z$ & -4.16 & 2.72 \\
\hline PBE0/aug3-cc-pVTZ & -3.64 & 2.82 \\
\hline X3LYP/aug3-cc-pVTZ & -3.60 & 2.86 \\
\hline B3LYP/aug3-cc-pVTZ & -3.18 & 2.84 \\
\hline BLYP/aug3-cc-pVTZ & -2.80 & 2.72 \\
\hline Experimental $^{\mathrm{a}}$ & $\begin{array}{c}-3.48 \\
(-4.3--3.2)\end{array}$ & \\
\hline $\begin{array}{c}\text { RRHO+RIMP2/aug-cc-pVTZ// } \\
\text { B3LYP/TZVP }\end{array}$ & -3.85 & \\
\hline $\begin{array}{c}\text { RRHO+RIMP2/aug-cc-pV(TQ)Z// } \\
\text { B3LYP/TZVP Level } \\
\text { (Extrapolations) }^{\mathrm{b}}\end{array}$ & -4.11 & \\
\hline
\end{tabular}


Table II. Vertical electron detachment energy (VDE) of negatively charged linear methanol dimers, trimers and tetramers as obtained at various levels of theory

\begin{tabular}{|c|c|c|c|}
\hline \multirow{2}{*}{ Level of theory } & \multicolumn{3}{|c|}{$\mathrm{VDE} / \mathrm{meV}$} \\
\hline & Dimer $^{\mathrm{a}}$ & Trimer $^{b}$ & Tetramer ${ }^{b, c}$ \\
\hline LC-BLYP/6-31(1+3+)G(d) & -35 & -13 & 61 \\
\hline BHandHLYP/6-31(1+3+)G(d) & -32 & -14 & 86 \\
\hline$\omega B 97 X-D / 6-31(1+3+) G(d)$ & 16 & 35 & 100 \\
\hline PBE0/6-31(1+3+)G(d) & 74 & 105 & \\
\hline X3LYP/6-31(1+3+)G(d) & 63 & 93 & \\
\hline B3LYP/6-31(1+3+)G(d) & 83 & 122 & \\
\hline BLYP/6-31(1+3+)G(d) & 42 & 139 & \\
\hline LC-BLYP/aug3-cc-pVDZ & $-19(3)$ & $-5(10)$ & $58(62)$ \\
\hline BHandHLYP/aug3-cc-pVDZ & $-16(23)$ & $1(36)$ & $85(103)$ \\
\hline$\omega B 97 X-D / a u g 3-c c-p V D Z$ & $25(39)$ & $44(56)$ & $95(100)$ \\
\hline PBE0/aug3-cc-pVDZ & 82 & 116 & \\
\hline X3LYP/aug3-cc-pVDZ & 94 & 119 & \\
\hline B3LYP/aug3-cc-pVDZ & 96 & 148 & \\
\hline BLYP/aug3-cc-pVDZ & 124 & 183 & \\
\hline LC-BLYP/aug3-cc-pVTZ & $-18(1)$ & $-2(10)$ & $59(61)$ \\
\hline BHandHLYP/aug3-cc-pVTZ & $-9(32)$ & $6(40)$ & $87(-)$ \\
\hline$\omega B 97 X-D / a u g 3-c c-p V T Z$ & $30(43)$ & $47(56)$ & $97(100)$ \\
\hline PBE0/aug3-cc-pVTZ & 90 & 112 & \\
\hline X3LYP/aug3-cc-pVTZ & 90 & 128 & \\
\hline B3LYP/aug3-cc-pVTZ & 122 & 158 & \\
\hline BLYP/aug3-cc-pVTZ & 119 & 183 & \\
\hline $\mathrm{MP} 2 / 6-31(1+3+) \mathrm{G}(\mathrm{d})$ & -44 & -16 & [44] \\
\hline MP2/aug3-cc-pVDZ & $-22(1)$ & $-16(-4)$ & {$[46(50)]$} \\
\hline MP2/aug3-cc-pVTZ & - & $-12(-3)$ & {$[48(50)]$} \\
\hline $\begin{array}{r}\text { CCSD(T)/aug3-cc-pVDZ// } \\
\text { (LC-BLYP) }\end{array}$ & -13 & 6 & 69 \\
\hline $\begin{array}{r}\mathrm{CCSD}(\mathrm{T}) / \mathrm{aug} 3-\mathrm{cc}-\mathrm{pVDZ} / / \\
\text { (BHandHLYP) }\end{array}$ & -21 & -11 & 56 \\
\hline $\begin{array}{r}\text { CCSD(T)/aug4-cc-pVDZ// } \\
\text { (BHandHLYP) }\end{array}$ & -9 & 5 & 60 \\
\hline
\end{tabular}

${ }^{a}$ All dimers (except CCSD(T)) are optimized. The numbers in parenthesis show single point VDEs on the optimized geometries after adding a diffuse function to the corresponding basis sets (i.e. aug4-cc-pVDZ, aug4-cc-pVTZ). CCSD(T) dimers are single point computed on LC-BLYP/ aug3-cc-pvdz and BHandHLYP/ aug3-cc-pvdz optimized geometries.

${ }^{\mathrm{b}}$ Single point calculations on geometries obtained with the $6-31(1+3+) G(d)$ basis set. The numbers in parenthesis show single point VDEs on the $6-31(1+3+) \mathrm{G}(\mathrm{d})$ geometries after adding a diffuse function to the corresponding basis sets (i.e. aug4-cc-pVDZ, aug4-cc-pVTZ). 
${ }^{c}$ Due to the non-converging MP2/6-31(1+3+)G(d) tetramer geometry, MP2 tetramer VDEs are evaluated at the BHandHLYP/6-31(1+3+)G(d) optimized tetramer geometry. See numbers in []. 


\section{References}

${ }^{1}$ B. C. Garrett, D. A. Dixon, D. M. Camaioni, D. M. Chipman, M. A. Johnson, C. D. Jonah, G. A. Kimmel, J. H. Miller, T. N. Rescigno, P. J. Rossky, S. S. Xantheas, S. D. Colson, A. H. Laufer, D. Ray, P. F. Barbara, D. M. Bartels, K. H. Becker, K. H. Bowen, Jr., S. E. Bradforth, I. Carmichael, J. V. Coe, L. R. Corrales, J. P. Cowin, M. Dupuis, K. B. Eisenthal, J. A. Franz, M. S. Gutowski, K. D. Jordan, B. D. Kay, J. A. LaVerne, S. V. Lymar, T. E. Madey, C. W. McCurdy, D. Meisel, S. Mukamel, A. R. Nilsson, T. M. Orlando, N. G. Petrik, S. M. Pimblott, J. R. Rustad, G. K. Schenter, S. J. Singer, A. Tokmakoff, L.-S. Wang, C. Wittig, and T. S. Zwier, Chem. Rev. $\mathbf{1 0 5}, 355$ (2005).

${ }^{2}$ G. A. Olah, Angew. Chem. Int. Ed. 44, 2636 (2005).

${ }^{3}$ J. H. Baxendale and P. Wardman, J. Chem. Soc., Faraday Trans. 1 69, 584 (1973).

${ }^{4}$ a) D. F. Feng and L. Kevan, Chem. Rev. 80, 1 (1980); b) L. Kevan, Radiat. Phys. Chem. 17, 413 (1981); c) L. Kevan, Chem. Phys. Lett. 66, 578 (1979).

${ }^{5}$ F. Y. Jou and G. R. Freeman, Can. J. Chem. 57, 591 (1979); F. Y. Jou and G. R. Freeman, J. Phys. Chem. 81, 909 (1977).

${ }^{6}$ X. Shi, F. H. Long, H. Lu and K. B. Eisenthal, J. Phys. Chem. 99, 6917 (1995).

${ }^{7}$ P. K. Walhout, J. C. Alfano, Y. Kimura, C. Silva, P. Reid and P. F. Barbara, Chem. Phys. Lett. 232, 135 (1995).

${ }^{8}$ C. M. Stuart, M. J. Tauber and R. A Mathies, J. Phys. Chem. A 111, 8390 (2007).

${ }^{9}$ H. Shen, N. Kurahashi, T. Horio, K. Sekiguchi and T. Suzuki, Chem. Lett. 39, 668 (2010).

${ }^{10}$ T. Horio, H. Shen, S. Adachi and T. Suzuki, Chem. Phys. Lett. 535, 12 (2012). 
${ }^{11}$ A. Kammrath, J. R. R. Verlet, G. B. Griffin, and D. M. Neumark, J. Chem. Phys. 125, 171102 (2006).

${ }^{12}$ A. Kammrath, G. B. Griffin, J. R. R. Verlet, R. M. Young, and D. M. Neumark, J. Chem. Phys. 126, 244306 (2007).

${ }^{13}$ M. H. Elkins, H. L. Williams and D. M. Neumark, J. Chem. Phys. 142, 234501 (2015).

${ }^{14}$ R. M. Young and D. M. Neumark, Chem. Rev. 112, 5553 (2012).

${ }^{15}$ L. Mones, L. Turi and P. J. Rossky, J. Chem. Phys. 135, 084501 (2011).

${ }^{16}$ J. R. R. Verlet, A. E. Bragg, A. Kammrath, O. Cheshnovsky, and D. M. Neumark, Science 307, 93 (2005).

${ }^{17}$ L. Ma, K. Majer, F. Chirot, and B. von Issendorff, J. Chem. Phys. 131, 144303 (2009).

${ }^{18}$ L. Turi, J. Chem. Phys. 110, 10364 (1999).

${ }^{19}$ J. Zhu and R. I. Cukier, J. Chem. Phys. 98, 5679 (1993).

${ }^{20}$ L. Turi, A. Mosyak, and P. J. Rossky, J. Chem. Phys. 107, 1970 (1997).

${ }^{21}$ A. Mosyak, P. J. Rossky, and L. Turi, Chem. Phys. Lett. 282, 239 (1998).

${ }^{22}$ A. Mosyak, O. V. Prezhdo, and P. J. Rossky, J. Mol. Struct. 485-486, 545 (1999).

${ }^{23}$ P. Mináry, L. Turi, and P. J. Rossky, J. Chem. Phys. 110, 10953 (1999).

${ }^{24}$ L. Turi, P. Mináry, and P. J. Rossky, Chem. Phys. Lett. 316, 456 (2000).

${ }^{25}$ L. Turi and P. J. Rossky, J. Chem. Phys. 120, 3688 (2004).

${ }^{26}$ L. Mones and L. Turi, J. Chem. Phys. 132, 154507 (2010).

${ }^{27}$ D. Borgis, P. J. Rossky and L. Turi, J. Chem. Phys. 125, 064501 (2006).

${ }^{28}$ L. Mones, P. J. Rossky and L. Turi, J. Chem. Phys. 133, 144510 (2010).

${ }^{29}$ J. M. Herbert, and M. Head-Gordon, J. Phys. Chem. A 109, 5217 (2005). 
${ }^{30}$ J. M. Herbert, and M. Head-Gordon, Phys. Chem. Chem. Phys. 8, 68 (2006).

${ }^{31}$ J. M. Herbert, Reviews in Computational Chemistry 28, ed. by A. L. Parrill and K. B. Lipkowitz, pp. 391-517 (2015).

${ }^{32}$ V. P. Vysotskiy, L. S. Cederbaum, T. Sommerfeld, V. K. Voora, and K. D. Jordan, J. Chem. Theory Comput. 8, 893 (2012).

${ }^{33}$ L. Turi, J. Chem. Phys. 144, 154311 (2016).

${ }^{34}$ L. Turi, J. Chem. Theory Comput. 11, 1745 (2015).

${ }^{35}$ F. A. Webster, P. J. Rossky and R. A. Friesner, Comput. Phys. Commun. 63, 494 (1991).

${ }^{36}$ W. L. Jorgensen, D. S. Maxwell, and J. Tirado-Rives, J. Am. Chem. Soc. 118, 11225 (1996).

${ }^{37}$ J. A. Pople, M. Head-Gordon, and K. Raghavachari, J. Chem. Phys., 87, 5968 (1987).

${ }^{38}$ C. Møller and M. S. Plesset, Phys. Rev. 46, 0618 (1934).

${ }^{39}$ A. D. Becke, Phys. Rev. A 38, 3098 (1988).

${ }^{40}$ C. Lee, W. Yang, and R. G. Parr, Phys. Rev. B 37, 785 (1988).

${ }^{41}$ H. Iikura, T. Tsuneda, T. Yanai, and K. Hirao, J. Chem. Phys. 115, 3540 (2001).

${ }^{42}$ A. D. Becke, J. Chem. Phys. 98, 5648 (1993).

${ }^{43}$ P. J. Stephens, J. F. Devlin, C. F. Chabalowski, and M. J. Frisch, J. Phys. Chem. 98, 11623 (1994).

${ }^{44}$ A. D. Becke, J. Chem. Phys. 98, 1372 (1993).

${ }^{45}$ J. C. Rienstra-Kiracofe, G. S. Tschumper, H. F. Schaefer, S. Nandi, and G. B. Ellison, Chem. Rev. 102, 231 (2002).

${ }^{46}$ J. P. Perdew, K. Burke, and M. Ernzerhof, Phys. Rev. Lett. 77, 3865 (1996).

${ }^{47}$ C. Adamo and V. Barone, J. Chem. Phys., 1106158 (1999). 
${ }^{48}$ X. Xu and W. A. Goddard III, Proc. Natl. Acad. Sci. USA 1012673 (2004).

${ }^{49}$ Y. Zhao and D. G. Truhlar, Theor. Chem. Acc 120215 (2008).

${ }^{50}$ J.-D. Chai, and M. Head-Gordon, Phys. Chem. Chem. Phys 106615 (2008)

${ }^{51}$ P. L. Silvestrelli, and M. Parrinello, J.Chem.Phys. 111, 3572 (1999).

${ }^{52}$ D. Svozil, and P. Jungwirth, J.Phys.Chem A 110, 9194 (2006).

${ }^{53}$ R. M. Richard, and J. M. Herbert, J. Chem. Theory Comput. 7, 1296 (2011).

${ }^{54}$ R. Baer, E. Livshits, and U. Salzner, Annu. Rev. Phys. Chem. 61, 85 (2010).

${ }^{55}$ M. Head-Gordon, J. A. Pople, and M. J. Frisch, Chem. Phys. Lett. 153503 (1988).

${ }^{56}$ W. Hehre, R. Ditchfield, and J. A. Pople, J. Chem. Phys. 56, 2257 (1972). M. J. Frisch, J. A. Pople, and J. S. Binkley, J. Chem. Phys. 803265 (1984).

${ }^{57}$ T. H. Dunning Jr., J. Chem. Phys. 901007 (1989). R. A. Kendall, T. H. Dunning Jr., and R. J. Harrison, J. Chem. Phys. 966796 (1992).

${ }^{58}$ Gaussian 09, Revision B.01, M. J. Frisch, G. W. Trucks, H. B. Schlegel, G. E. Scuseria, M. A. Robb, J. R. Cheeseman, G. Scalmani, V. Barone, B. Mennucci, G. A. Petersson, H. Nakatsuji, M. Caricato, X. Li, H. P. Hratchian, A. F. Izmaylov, J. Bloino, G. Zheng, J. L. Sonnenberg, M. Hada, M. Ehara, K. Toyota, R. Fukuda, J. Hasegawa, M. Ishida, T. Nakajima, Y. Honda, O. Kitao, H. Nakai, T. Vreven, J. A. Montgomery, Jr., J. E. Peralta, F. Ogliaro, M. Bearpark, J. J. Heyd, E. Brothers, K. N. Kudin, V. N. Staroverov, R. Kobayashi, J. Normand, K. Raghavachari, A. Rendell, J. C. Burant, S. S. Iyengar, J. Tomasi, M. Cossi, N. Rega, J. M. Millam, M. Klene, J. E. Knox, J. B. Cross, V. Bakken, C. Adamo, J. Jaramillo, R. Gomperts, R. E. Stratmann, O. Yazyev, A. J. Austin, R. Cammi, C. Pomelli, J. W. Ochterski, R. L. Martin, K. Morokuma, V. G. Zakrzewski, G. A. Voth, P. Salvador, J. J. Dannenberg, S. Dapprich, A. D. Daniels, Ö. Farkas, J. B. Foresman, J. V. Ortiz, J. Cioslowski, and D. J. Fox, Gaussian, Inc., Wallingford CT, 2009. 
${ }^{59}$ CFOUR, a quantum chemical program package written by J.F. Stanton, J. Gauss, M.E. Harding, P.G. Szalay with contributions from A.A. Auer, R.J. Bartlett, U. Benedikt, C. Berger, D.E. Bernholdt, Y.J. Bomble, L. Cheng, O. Christiansen, M. Heckert, O. Heun, C. Huber, T.-C. Jagau, D. Jonsson, J. Jusélius, K. Klein, W.J. Lauderdale, D.A. Matthews, T. Metzroth, L.A. Mück, D.P. O'Neill, D.R. Price, E. Prochnow, C. Puzzarini, K. Ruud, F. Schiffmann, W. Schwalbach, C. Simmons, S. Stopkowicz, A. Tajti, J. Vázquez, F. Wang, J.D. Watts and the integral packages MOLECULE (J. Almlöf and P.R. Taylor), PROPS (P.R. Taylor), ABACUS (T. Helgaker, H.J. Aa. Jensen, P. Jørgensen, and J. Olsen), and ECP routines by A. V. Mitin and C. van Wüllen. For the current version, see http://www.cfour.de.

${ }^{60}$ L. A. Curtiss, M. Blander, Chem. Rev. 88, 827 (1988).

${ }^{61}$ M. Umer, and K. Leonhard, J. Phys. Chem. A 117, 1569 (2013).

${ }^{62}$ B. H. Abdoul-Carime, and C. Desfrançois, Eur. Phys. J. D 2149 (1998).

${ }^{63}$ N. I. Hammer, J-W. Shin, J. M. Headrick, E. G. Diken, J. R. Roscioli, G. H. Weddle, and M. A. Johnson, Science 306, 675 (2004).

${ }^{64}$ N. I. Hammer, J. R. Roscioli, J. C. Bopp, J. M. Headrick, and M. A. Johnson, J. Chem. Phys. 123, 244311 (2006).

${ }^{65}$ G. Makov, and A. Nitzan, J. Phys. Chem. 98, 3459 (1994).

${ }^{66}$ F. Uhlig, J. M. Herbert, M. P. Coons, and P. Jungwirth, J. Phys. Chem. A 118, 7507 (2014).

${ }^{67}$ M. Feyereisen, G. Fitzgerald, and A. Komornicki, Chem. Phys. Lett. 208, 359 (1993).

${ }^{68}$ F. Weigend and M. Haser, Theor. Chem. Acc. 97, 331 (1997). 